\title{
miR-125a-3p targetedly regulates GIT1 expression to inhibit osteoblastic proliferation and differentiation
}

\author{
XIAO-MEI TU ${ }^{*}$, YANG-LIN GU ${ }^{2 *}$ and GUO-QIN REN ${ }^{1}$ \\ Departments of ${ }^{1}$ Nursing and ${ }^{2}$ Orthopedics, Wuxi Second Hospital Affiliated to Nanjing Medical University, \\ Wuxi, Jiangsu 214002, P.R. China
}

Received July 30, 2015; Accepted September 22, 2016

DOI: $10.3892 /$ etm.2016.3874

\begin{abstract}
Osteoblasts are a prerequisite for osteogenesis and bone formation, and play a key role in metabolic balance, growth, development and wound repair. $\mathrm{G}$ protein-coupled receptor kinase interacting protein 1 (GIT1) and a series of miRNAs are known to have important effects in the growth and migration of osteoblasts, but little is known about micro RNAs (miRNAs) targeting GIT1. The present study found that miR-125a-3p has matching sites on GIT1. In the osteoblastic differentiation process of human bone marrow-derived mesenchymal stem cells (HMSCs), the expression of miR-125a-3p was suppressed compared with that in non-differentiating (HMSCs) while the expression of GIT1 showed a gradual and significant increase. Thus, miR-125a-3p expression was negatively correlated with the expression of GIT1. Following the transfection of human osteoblasts with miR-125a-3p mimics and inhibitors, respectively, the effect on GIT1 expression was opposite to the change of miR-125a-3p expression. In addition, the impact of miR-125a-3p and GIT1 on osteoblastic proliferation and differentiation was detected, and the results indicated that miR-125a-3p targetedly regulated GIT1 expression to inhibit osteoblastic proliferation and differentiation. These findings may provide a theoretical basis for clarifying the physiological and pathological role of miRNAs in osteoblast differentiation and maturation processes, and for the physiological and pathological investigation of bone.
\end{abstract}

\section{Introduction}

Osteoblasts, which are amongst the most important cells in the bone development and remodeling process, are responsible

Correspondence to: Dr Guo-Qin Ren, Department of Nursing, Wuxi Second Hospital Affiliated to Nanjing Medical University, 68 Zhongshan Road, Wuxi, Jiangsu 214002, P.R. China

E-mail: gqren15@126.com

*Contributed equally

Key words: G protein-coupled receptor kinase interacting protein 1, miR-125-3p, osteoblast, microRNA for the synthesis, secretion and mineralization of bone matrix and have an important effect in bone development, maturation, repair and remodeling $(1,2)$. Mesenchymal stem cells (MSCs) first differentiated into osteoprogenitor cells, then into preosteoblasts, osteoblasts, and finally into osteocytes (3).

$\mathrm{G}$ protein-coupled receptor kinase interacting protein 1 (GIT1) is widely present in mammals and birds and exists mainly in the focal adhesion and cytoplasmic complex structure of cells (4). It plays an important role in cell growth and migration through interaction with a variety of proteins, including Rac1, p21-activated kinase, phospholipase C and paxillin (5). A previous study has indicated that GIT1 can be expressed in both osteoblasts and osteoclasts (6), suggesting that GIT1 may be involved in the bone metabolic process. Cell spreading and Boyden chamber assays have shown that GIT1 (Y321F) mutant inhibits the motility and migration of osteoblasts induced by platelet-derived growth factor (PDGF), and it has been demonstrated that phosphorylation of GIT1 tyrosine 321 is required for association with focal adhesion kinase at focal adhesions and for PDGF-activated migration of osteoblasts (7). Therefore, GIT1 possesses a vital function in osteoblasts.

MicroRNA (miRNA), an endogenous non-coding single-stranded small RNA, exists extensively in the biological genome. It is able to pair with and bind to 3 ' untranslated regions (3'UTRs) of the targeted mRNA incompletely to degrade mRNA or repress the translation of mRNA, and is involved in regulating more than half of all gene expression (8). In addition, miRNA is an important post-transcriptional regulatory factor and plays an extensive and important role in cell proliferation, differentiation, apoptosis, tissues development, oncogenesis and other physiological processes (9). Studies have shown that miRNAs also have a significant regulatory effect on osteoblastic differentiation and bone development $(10,11)$. In the osteoblast differentiation process, miRNA closely associated with this process, known as 'osteomiRs', are present, which can regulate the differentiation process via many pathways $(11,12)$. For example, in the late stage of osteoblast differentiation, which is the bone matrix deposition and mineralization phase, miR-29a and miR-29c inhibit the expression of the marker gene osteonectin so as to regulate the bone remodeling process (13).

Nevertheless, studies concerning miRNAs targeting GIT1 are rare and, to the best of our knowledge, no studies have involved miRNAs and GIT1 in osteoblasts. Given the impor- 
tant role of GIT1, the present study aimed to investigate the regulatory relationship and the interaction between GTI1 and relevant miRNA in osteoblasts.

\section{Materials and methods}

Cell culture and osteoblastic differentiation. Bone marrow-derived human MSCs (HMSCs) and human osteoblasts were purchased from ScienCell Research Laboratories, Inc. (Carlsbad, CA, USA). HMSCs were cultured in mesenchymal stem cell medium (MSCM; cat. no. 7501; ScienCell Research Laboratories, Inc.), and osteoblasts were cultured in osteoblast medium (ObM; cat. no. 4601; ScienCell Research Laboratories, Inc.). Osteoblastic differentiation was induced by changing to media containing $10 \%$ FBS supplemented with $100 \mathrm{ng} / \mathrm{ml}$ bone morphogenetic protein 2 (BMP-2; R\&D Systems, Inc., Minneapolis, MN, USA).

Overexpression vector construction and transfection. TRIzol (Thermo Fisher Scientific, Inc., Waltham, MA, USA) was used to extract RNA from osteoblasts according to the instructions of the manufacturer. Following this, the RNA was subjected to reverse transcription-polymerase chain reaction (RT-PCR) to amplify the coding region of GIT1 using an Advantage RT for PCR kit (Takara Biotechnology Co., Ltd., Dalian, China). The product was digested with KpnI and EcoRI (Takara Biotechnology Co., Ltd.), and cloned into pcDNA3.1 vectors (Thermo Fisher Scientific, Inc.), sequenced and verified in a 3730xl DNA Analyzer (Applied Biosystems; Thermo Fisher Scientific, Inc.). The primers used for GIT1 amplification are shown in Table I. Transfection of human osteoblasts was carried out using Lipofectamine 2000, following the instructions of the manufacturer (Thermo Fisher Scientific, Inc.,) in 6-well cell culture plates when the cell confluence reached $\sim 70 \%$. The concentration of GIT1 overexpression vector for transfection was $4 \mu \mathrm{g} /$ well, and that of human GIT1 small interfering RNA (siRNA; sc-35,477; Santa Cruz Biotechnology, Inc., Dallas, TX, USA), miR-125a-3p mimics and inhibitors (Shanghai GenePharma Co., Ltd., Shanghai, China) was $50 \mathrm{nM} /$ well. For osteoblastic differentiation, the medium was replaced $4 \mathrm{~h}$ after transfection with fresh medium containing $10 \% \mathrm{FBS}$ and 100 ng/ml BMP-2.

RT-quantitative PCR ( $q P C R$ ). Total RNA was isolated from cultured cell samples using TRIzol according to the manufacturer's instructions, and a mirVana miRNA Isolation kit (Applied Biosystems; Thermo Fisher Scientific, Inc.) was used to purify the miRNAs. Expression levels were measured by RT-qPCR. First, cDNA was synthesized by RT using random and oligo-dT primers or specific primers for miRNA-125a-3p and the GoScript Reverse Transcription System (Promega Corporation, Shanghai, China). Thermal cycling was performed as follows: one cycle of $95^{\circ} \mathrm{C}$ for $5 \mathrm{~min}$ and 40 cycles of $95^{\circ} \mathrm{C}$ for $30 \mathrm{sec}, 55^{\circ} \mathrm{C}$ for $30 \mathrm{sec}$ and $72^{\circ} \mathrm{C}$ for $30 \mathrm{sec}$. qPCR was conducted using the GoTaq qPCR Master mix (Promega Corporation) with an ABI PRISM ${ }^{\circledR} 7500$ Sequence Detection system (Applied Biosystems) following the manufacturer's instructions. The reaction was performed at one cycle of $95^{\circ} \mathrm{C}$ for $2 \mathrm{~min}$, followed by 40 cycles of $95^{\circ} \mathrm{C}$ for $15 \mathrm{sec}$ and $60^{\circ} \mathrm{C}$ for $32 \mathrm{sec}$. For measurement of miRNA expression, specific primers for miRNA-125a-3p and U6 were used. The primer sequences are shown in Table I. Three independent experiments were conducted for each sample. Data were analyzed using the $2^{-\Delta \Delta \mathrm{Cq}}$ method (14).

Western blot analysis. Total cellular proteins were extracted by incubating cells in radioimmunoprecipitation assay (RIPA) buffer (sc-24948; Santa Cruz Biotechnology, Inc.), separating the proteins (50 $\mu \mathrm{g}$ per lane) by $10 \%$ SDS-PAGE and transferring them to nitrocellulose membranes (Bio-Rad Laboratories, Inc., Shanghai, China). The membranes were blocked with 5\% non-fat milk for $1 \mathrm{~h}$ and then incubated with GIT1 (cat. no. 2919; rabbit anti-human polyclonal antibody; 1:1,000; Cell Signaling Technology, Inc., Beverly, MA, USA) or GAPDH (14C10) rabbit monoclonal antibody (cat. no. 2118; rabbit anti-human monoclonal antibody; 1:1,000; Cell Signaling Technology, Inc.) in $5 \%$ non-fat milk overnight at $4^{\circ} \mathrm{C}$. Immunoreactive proteins were visualized using horseradish peroxidase (HRP)-conjugated secondary antibodies (cat. no. 7074; anti-rabbit IgG, HRP-linked antibody; 1:7,000-8,000 Cell Signaling Technology, Inc.) and enhanced chemiluminescence reagents (Pierce; Thermo Fisher Scientific, Inc.). Images were analyzed using Image-Pro Plus 6.0 software (Media Cybernetics, Inc., Rockville, MD, USA). Each band was scanned with background correction, and values were averaged and expressed as the mean \pm standard deviation (SD).

Dual luciferase assay. GIT1 3'UTR was cloned into the dual-luciferase reporter vector psiCHECK-2 (Promega Corporation), and the seed region of the miR-125a-3p binding site in the GIT1 3'UTR was mutated using a QuikChange II Site-Directed Mutagenesis kit (Stratagene; Agilent Technologies, Inc., Santa Clara, CA, USA). For luciferase assay, HEK293 cells (American Type Culture Collection, Manassas, VA, USA) were transfected at $80 \%$ confluency in 24-well dishes with psiCHECK-2 (with GIT1 3'UTR or GIT1 3'UTR mutation), and miR-125a-3p mimics by Lipofectamine 2,000. Cells were analyzed at $24 \mathrm{~h}$ post-transfection. Firefly and Renilla luciferase activities were quantified in lysates using the Dual Luciferase Reporter Assay kit (Promega Corporation) on a Glomax 20/20 luminometer (Promega Corporation) according to the manufacturer's recommendations. Luciferase readings were corrected for background and firefly luciferase values were normalized to Renilla to control for transfection efficiency. All assays were performed in triplicate in three independent experiments.

Immunoprecipitation. HEK293 cells were lysed in RIPA buffer containing protease inhibitors (Roche Diagnostics, Basel, Switzerland) and centrifuged at $10,000 \mathrm{x} g$ for $10 \mathrm{~min}$ at $4^{\circ} \mathrm{C}$. For immunoprecipitation, the lysates were incubated with monoclonal anti-argonaute 2 (Ago2) antibody (1:50; cat. no. 2897; Cell Signaling Technology, Inc.) overnight at $4^{\circ} \mathrm{C}$. Subsequently, $5 \mathrm{ml}$ hybridoma was coupled to $80 \mu \mathrm{l}$ Protein-G-Sepharose (GE Healthcare Life Sciences, Chalfont, UK). Beads were subsequently incubated with $10 \mathrm{ml} \mathrm{HEK} 293$ lysate for $5 \mathrm{~h}$ under constant rotation at $4^{\circ} \mathrm{C}$. After incubation, the beads were washed three times with washing buffer. Finally, the beads were washed once with PBS. Co-precipitated RNA was extracted using phenol:chloroform:isoamyl alcohol 
Table I. Sequences of primers.

\begin{tabular}{lll}
\hline Primer set & \multicolumn{1}{c}{ Sense (5'-3') } & Antisense (5'-3') \\
\hline GIT1 CDS amplification & GGGGTACCGCCACCATGTCCC & CGGAATTCTCACTGCTTCTTC \\
& GAAAGGGGCCG & TCTCGGGTG \\
Quantitative PCR & & \\
GAPDH & GGTATCGTGGAAGGACTC & GTAGAGGCAGGGATGATG \\
GIT1 & ATGTATGAACCTGGCTCTG & TGAATAGATGGCGTCGTC \\
Runx2 & CAAGGACAGAGTCAGATTAC & GTGGTAGAGTGGATGGAC \\
Osterix & TGCTTGAGGAGGAAGTTC & CTTTGCCCAGAGTTGTTG \\
GIT1 3'UTR amplification & CCGCTCGAGCCTCTCTCCCCA & ATAAGAATGCGGCCGCTAACA \\
& CACCCTCA & GCTCATGGTCACTTCTTTAT \\
\hline Underlining and bold denotes restriction enzyme cutting sites. GIT1, G protein-coupled receptor kinase interacting protein 1; CDS, coding \\
sequence; PCR, polymerase chain reaction; UTR, untranslated region. & \\
\hline
\end{tabular}

(25:24:1; cat. no. 15593-031; Invitrogen; Thermo Fisher Scientific, Inc.). The RNA pellet was used for RT-qPCR.

5-Bromo-2'-deoxyuridine (BrdU) cell proliferation assay. BrdU assay was used to investigate the effects of miR-125a-3p and GIT1 on cell proliferation. Briefly, cultured cells were seeded into a 6-well plate and incubated for $24 \mathrm{~h}$. Transfections with miR-125a-3p mimics and inhibitors, plasmids and siRNAs were then carried out using Lipofectamine 2000. A BrdU Cell Proliferation Assay kit (cat. no. 6813; Cell Signaling Technology, Inc.) was used for the quantification of cell proliferation according to the protocol provided by the manufacturer. Cell proliferation was expressed as a mean percentage of that of the control (set at 100\%).

Alkaline phosphatase (ALP) measurement. Cells were washed twice and then lysed on ice for $30 \mathrm{~min}$ in lysis buffer composed of Mammalian Protein Extraction Reagent and Protease Inhibitor Cocktail (CW Biotech Co., Beijing, China). In the following step, lysates were collected and centrifuged at $10,000 \times \mathrm{g}$ for $10 \mathrm{~min}$, and the supernatants were transferred into a new tube for the detection of ALP activity. The activity of ALP was measured using a commercially available Alkaline Phosphatase Assay kit (Nanjing Jiancheng Bioengineering Institute, Nanjing, China). Supernatants were also analyzed for protein concentration using a bicinchoninic acid (BCA) assay kit (Beyotime Institute of Biotechnology, Haimen, China), and ALP activity was normalized for total protein concentration. The absorbance values at 490 and $570 \mathrm{~nm}$, respectively for ALP and BCA analysis, were detected with a Multifunctional Microplate Reader (Bio-Rad Laboratories, Inc.).

Enzyme-linked immunosorbent assay (ELISA). Osteocalcin (OC) secretions from culture supernatants were assessed using an ELISA kit (cat. no. KAQ1381; Invitrogen). A 25- $\mu$ l portion of each sample was added to the appropriate wells, and then $100 \mu \mathrm{l}$ working anti-OST-HRP conjugate was added to all wells and the plates were incubated for $2 \mathrm{~h}$ at room temperature. Solution was thoroughly aspirated or decanted from the wells and discarded, and the wells were then washed 3 times.
Chromogen solution $(100 \mu \mathrm{l})$ to each well, and the plate was incubated for $30 \mathrm{~min}$ at room temperature in the dark. Stop solution $(100 \mu \mathrm{l})$ was added to each well, and the absorbance of each well was read at $450 \mathrm{~nm}$.

Statistical analysis. The experiments were carried out at least in triplicate and results were expressed as mean \pm SD. SPSS statistical package (SPSS 17.0 for Windows; SPSS, Inc., Chicago, IL, USA) was used for statistical analysis. Differences were analyzed by non-parametric statistical analysis (Mann-Whitney U tests) between control and treated groups, and $\mathrm{P}<0.05$ was considered to indicate a statistically significant difference.

\section{Results}

Prediction and verification of candidate miRNAs of GIT1. The software applications miRanda, TargetScanHuman, miRBase and miRWalk were used to predict miRNAs targeting GIT1, and the results revealed that miR-125a-3p had good matching sites with the GIT1 3'UTR (Fig. 1A). miR-125b, another member of the miR-125 family, was considered to be associated with osteoblast differentiation on the basis of a previous study (15). It has been indicated to achieve a regulatory effect by inhibiting the expression of the cell proliferation-associated ErbB2 gene to suppress stromal cell line ST2 proliferation and then directly affect osteoblast differentiation $(15,16)$. Therefore, the regulatory relationship between miR-125a-3p and GIT1 in osteoblasts is worthy of further investigation.

First, a dual-luciferase reporter assay was used to verify whether there are binding sites of miR-125a-3p in GIT1. The software applications miRanda (microrna.org/microrna/getGeneForm.do), TargetScanHuman (targetscan.org/vert_71/),miRBase(mirbase.org/)andmiRWalk (zmf.umm.uni-heidelberg.de/apps/zmf/mirwalk2/) predicted that GIT1 has miR-125a-3p binding sites. GIT1 3'UTR was amplified (see Table I for primers) and cloned into the dual-luciferase reporter vector psiCHECK-2 and the potential target sites of miR-125a-3p were mutated (Fig. 1A). These two plasmids were then transfected into HEK293 cells together with miR-125a-3p mimics, respectively, in order to analyze the 
A

$$
\begin{aligned}
\text { hsa-miR-125a-3p: } & \text { 3' ccGAGGGUUCUUGGAGUGGACa 5' } \\
\text { GIT1 wt 3' UTR: } & \text { 5' cuCUCCC C ACACCCU CACCUGC 3' } \\
\text { GIT1 } \mu \text { 3' UTR: } & 5^{\prime} \text { cuCUCCC C ACACAGCUUAGCAC 3' }
\end{aligned}
$$

B

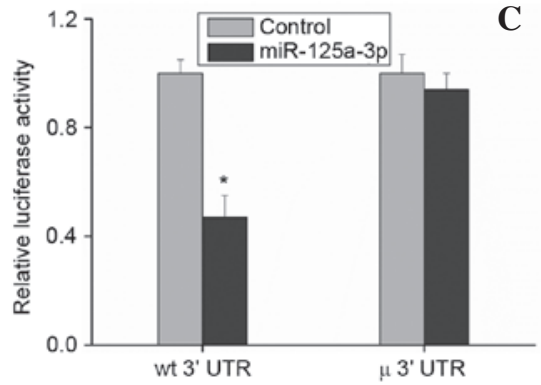

C

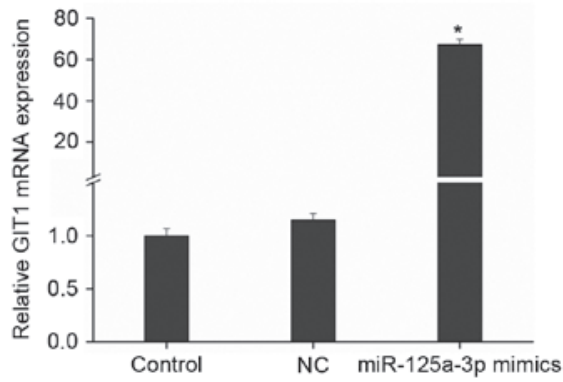

Figure 1. Prediction of miR-125a-3p gene targeting. (A) Predicted miR-125a-3p target sites in the conservative sequence of GIT1 3'UTR. (B) Luciferase expression levels of HEK293 cells transfected with GIT1 3'UTR or mutant GIT1 3'UTR vector plus miR-125a-3p mimics or control. (C) Monoclonal antibody anti-Ago2 was immobilized on Protein-G-Sepharose beads and incubated with HEK293 cell lysates after transfection with miR-125a-3p mimics, and then the co-immunoprecipitated Ago-bound RNAs were extracted and subjected to reverse transcription-quantitative polymerase chain reaction to detect GIT1 mRNA expression. Results are presented as the mean plus standard deviation from three samples (" $\mathrm{P}<0.01$ vs. control). miR, microRNA; GIT1, G protein-coupled receptor kinase interacting protein 1; UTR, untranslated region; Ago2, argonaute 2; wt, wild type; $\mu$, mutant.

A

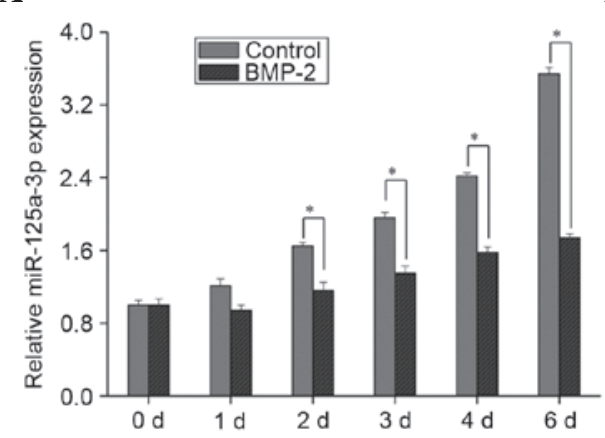

B

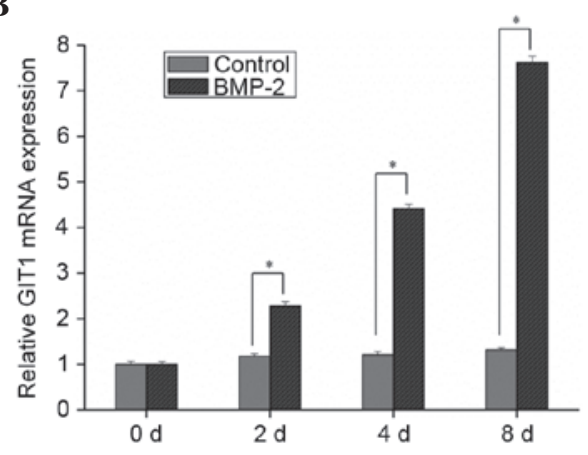

Figure 2. Relative expression of miR-125a-3p and GIT1 during osteoblastic differentiation. Human bone-marrow derived mesenchymal stem cells were harvested after 1, 2, 3, 4, 6 and 8 days after BMP-2 addition, and the expression levels of (A) miR-125a-3p and (B) GIT1 were measured. Results are presented as the mean plus standard deviation. "P<0.01 as indicated. Control, cells without BMP-2; BMP-2, cells treated with BMP-2; miR, microRNA; GIT1, G protein-coupled receptor kinase interacting protein 1; BMP, bone morphogenetic protein; d, days.

change of luciferase expression. Cells transfected with blank reporter vector were taken as the control. The cells transfected with the vector containing GIT1 3'UTR clones showed a reduction of $\sim 50 \%$ in luciferase expression level, with a statistically significant difference, $(\mathrm{P}<0.01 \mathrm{vs}$. control) while those transfected with the vector containing mutant GIT1 3'UTR clones had a non-significant change in the luciferase expression level (Fig. 1B). This illustrates that miR-125a-3p did have significant binding sites in the GIT1 3'UTR, suggesting that GIT1 is a target gene of miR-125a-3p.

Ago2 is a core component of the RNA-induced silencing complex, associating with both miRNAs and their mRNA targets (17). Thus, immunopurifying Ago2 under the appropriate conditions might retain associated miRNAs and mRNAs, allowing miRNA targets to be identified. Therefore, monoclonal anti-Ago2 antibody was immobilized on Protein-G-Sepharose beads and incubated with HEK293 cell lysates; then the co-immunoprecipitated Ago-bound RNAs were extracted and subjected to RT-qPCR to detect GIT1 mRNA expression. The results shown in Fig. $1 \mathrm{C}$ indicate that GIT1 mRNA expression increased significantly following transfection with miR-125a-3p mimics. This further illustrates that miR-125a-3p can targetedly regulate GIT1.

Association between the expression of $m i R-125 a-3 p$ and GIT1 in osteoblast differentiation. miR-125b is associated with osteoblast differentiation (15), but it is unclear whether miR-125a-3p is also associated with osteoblast differentiation. Thus, further investigation of this is necessary. After adding BMP-2 to HMSCs for induction of differentiation, the changes of miR-125a-3p and GIT1 expression were detected. According to the results, when HMSCs were not induced to differentiate, as the confluence tended to $100 \%$, the expression of miR-125a-3p increased gradually (Fig. 2A). However, while HMSCs were differentiated to osteoblasts, the expression of miR-125a-3p also increased over time, but the levels of increase were significantly suppressed ( $\mathrm{P}<0.01$; Fig. $2 \mathrm{~A})$, which indicated that miR-125a-3p expression was reduced as osteoblast differentiation proceeded. Moreover, the expression level of GIT1 was not significantly changed when HMSCs were in normal culture; however, when BMP-2 was added to induce osteoblast differentiation, GIT1 expression gradually increased 
A

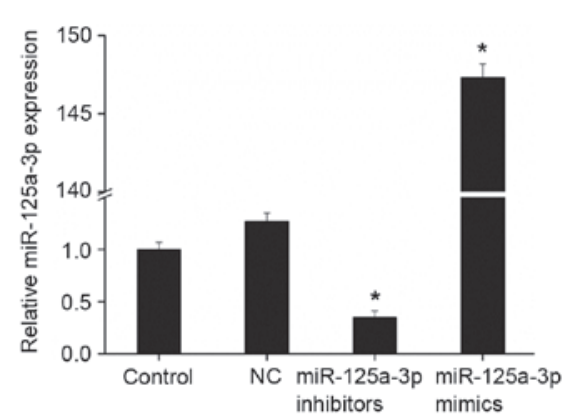

C

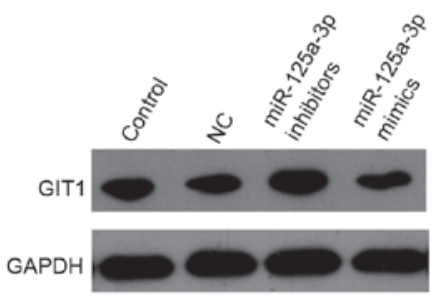

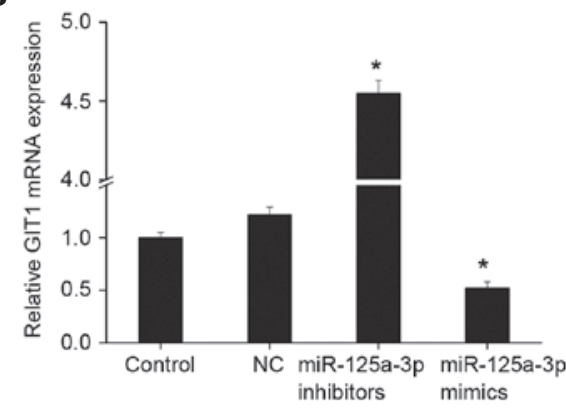

D

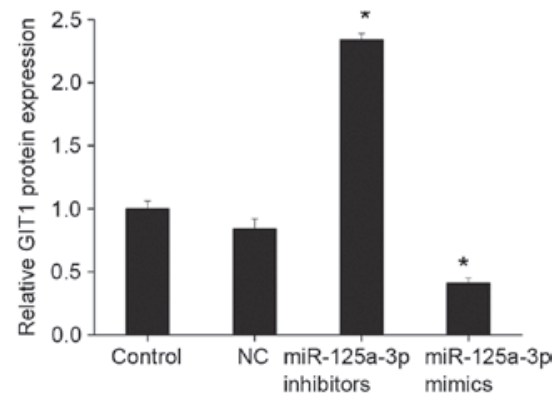

Figure 3. GIT1 and miR-125a-3p expression in osteoblasts. Human osteoblasts were transfected with miR-125a-3p mimics or inhibitors, and then the expression of (A) miR-125a-3p and (B) GIT1 was measured by reverse transcription-quantitative polymerase chain reaction. The protein expression of GIT1 was also examined by western blot analysis; (C) a representative western blot and (D) quantified expression levels are shown. Experiments were carried out at least in triplicate and the results are expressed as the mean plus standard deviation. ${ }^{*} \mathrm{P}<0.01$ vs. control. miR, microRNA; GIT1, G protein-coupled receptor kinase interacting protein 1; NC, negative control (pcDNA3.1 blank vector).

as the induction of differentiation proceeded (Fig. 2B). From the aforementioned results, in the osteoblastic differentiation process, miR-125a-3p expression was negatively correlated with GIT1 expression, and upregulation of GIT1 expression may be caused by the inhibition of miR-125a-3p expression.

miR-125a-3p downregulates GIT1 expression in osteoblasts. On the basis of the aforementioned results, it may be speculated that miR-125a-3p regulates GIT1. miR-125a-3p mimics and inhibitors were transfected into human osteoblasts, and fluorescence qPCR was used to detect the expression of miR-125a-3p and GIT1. As shown in Fig. 3A, when mimics were transfected, the expression level of miR-125a-3p was significantly upregulated, while the expression of miR-125a-3p was significantly inhibited following the addition of miR-125a-3p inhibitors (both $\mathrm{P}<0.01$ ). The expression of GIT1 was downregulated with upregulation of miR-miR-125a-3p expression, as shown by fluorescence qPCR and western blotting (Fig. 3B-D). By contrast, GIT1 expression increased markedly when miR-125a-3p expression was inhibited. This suggests that miR-125a-3p targetedly regulates the expression of GIT1 in osteoblasts.

miR-125a-3p affects osteoblast proliferation and differentiation by regulating GIT1 expression. The impact of miR-125a-3p and GIT1 on osteoblast proliferation and differentiation was investigated. The proliferation of osteoblasts transfected with miR-125a-3p mimics declined significantly compared with the control while the proliferation of those transfected with miR-125a-3p inhibitors increased (both $\mathrm{P}<0.01$; Fig. 4A). This suggests that miR-125a-3p suppresses osteoblast proliferation. The proliferation of osteoblasts transfected with GIT1 overexpression vector was increased markedly, while their proliferation was inhibited following transfection with GIT1 siRNA to reduce GIT1 expression (Fig. 4A), which indicates that GIT1 promotes osteoblast proliferation. In addition, following co-transfection with miR-125a-3p mimics and GIT1 overexpression vector, upregulation of GIT1 expression blocked the proliferation-suppressing effect of miR-125a-3p, and the osteoblast proliferation was increased; a significant difference was found compared with the miR-125a-3p mimics transfection group (Fig. 4A). This indicates that miR-125a-3p can targetedly regulate GIT1 expression to inhibit osteoblast proliferation.

Further analysis of osteoblast differentiation indicates that the upregulation of miR-125a-3p expression reduced ALP activity (Fig. 4B), OC secretion (Fig. 5A) and the expression of Runx2 and osterix (Fig. 5B). That is, miR-125a-3p had an inhibitory effect on osteoblast differentiation, and downregulation of its expression promoted osteoblast differentiation. GIT1 promoted osteoblast differentiation, although not very strongly (Figs. 4B and 5). Following co-transfection with miR-125a-3p mimics and GIT1 overexpression vector, the inhibitory effect of miR-125a-3p on osteoblast differentiation was weakened (Figs. 4B and 5). Hence, in osteoblasts, it may be concluded that miR-125a-3p affects the proliferation and differentiation of osteoblasts by regulating the expression of GIT1.

\section{Discussion}

Osteoblasts play an important role in bone formation, development, remodeling and repair processes, and 
A

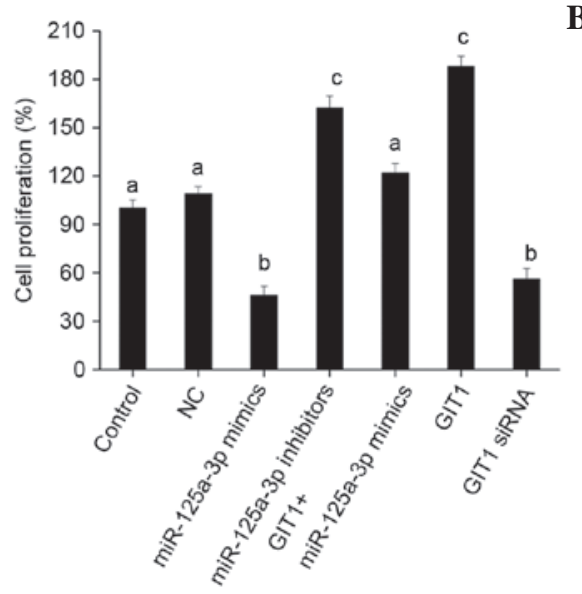

B

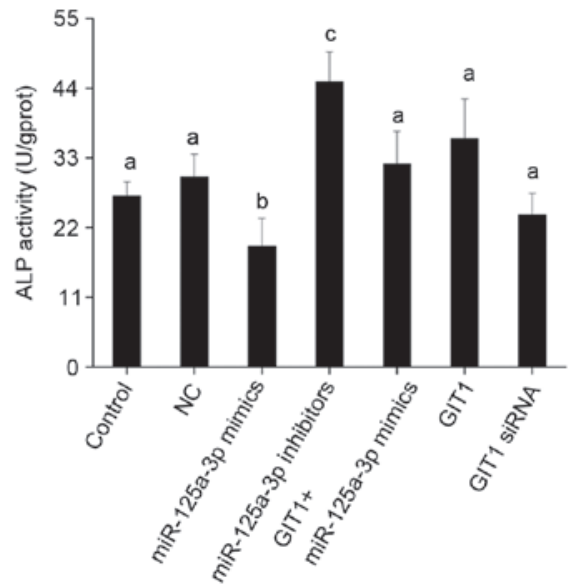

Figure 4. Roles of miR-125a-3p and GIT1 in osteoblast proliferation and ALP activity assays. Osteoblasts were transfected with GIT1-overexpressing vector and/or miR-125a-3p mimics, miR-125a-3p inhibitors or GIT1 siRNA, and cultured for $72 \mathrm{~h}$. (A) Cell proliferation was examined by 5-bromo-2'-deoxyuridine assay. (B) ALP activity was determined in cell lysates and normalized to protein content. Results are presented as mean plus standard deviation (n=3). Bars with different letter labels are significantly different at the $\mathrm{P}<0.01$ level. miR, microRNA; GIT1, G protein-coupled receptor kinase interacting protein 1; ALP, alkaline phosphatase; siRNA, small interfering RNA; NC, negative control (pcDNA3.1 blank vector).

A

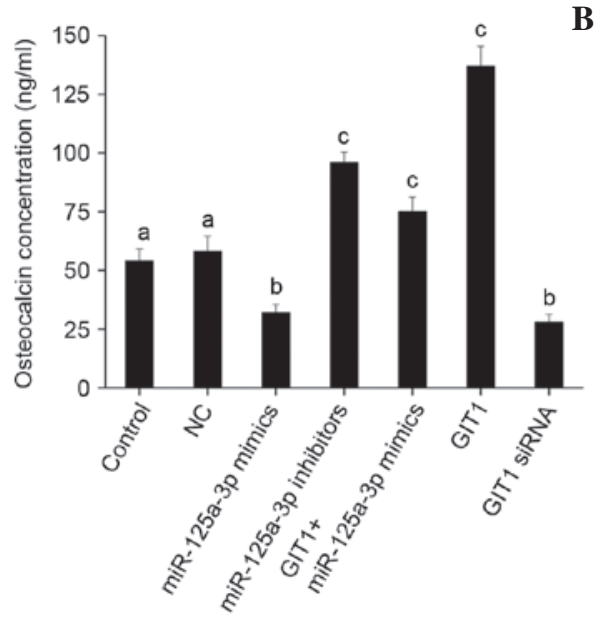

B

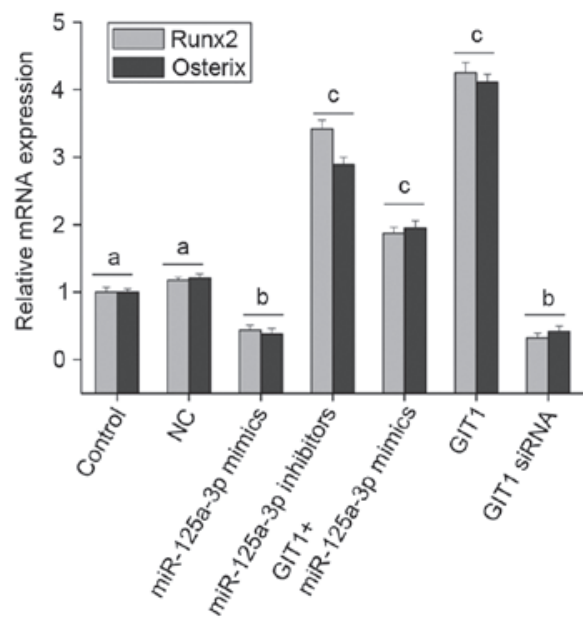

Figure 5. OC secretion, and the mRNA expression of Runx 2 and osterix. Osteoblasts were transfected with GIT1-overexpressing vector and/or miR-125a-3p mimics, miR-125a-3p inhibitors and GIT1 siRNA, and cultured for $72 \mathrm{~h}$. (A) OC secretion was assessed from culture supernatants by enzyme-linked immunosorbent assay. (B) Expression of Runx 2 and osterix was measured by reverse transcription-quantitative polymerase chain reaction. Results are presented as the mean plus standard deviation from three independent experiments. Bars with different letter labels are significantly different at the P $<0.01$ level. OC, osteocalcin; Runx2, runt-related transcription factor 2; miR, microRNA; GIT1, G protein-coupled receptor kinase interacting protein 1; siRNA, small interfering RNA; NC, negative control (pcDNA3.1 blank vector).

various growth factors may affect osteoblast proliferation, differentiation and extracellular matrix synthesis (18).

In recent years, the relationship between miRNAs and bone formation has attracted increasing attention. Notably, the knockout of Dicer and Drosha leads to generation failure of all miRNAs in cells, thus causing a loss of differentiation capacity of bone marrow stroma stem cells into osteoblasts and adipocytes (19). This suggests that an appropriate expression level of miRNAs is essential for the osteoblastic differentiation of stem cells. In addition, numerous studies have shown that GIT1 plays a significant role in the growth and migration of osteoblasts (6-7,20). Consequently, investigation of the effect of GIT1 and relevant miRNAs in osteoblasts is worthwhile. However, studies of miRNAs targeting GIT1 are rare at present, with only a few relevant reports in tumors $(21,22)$.
In the present study, prediction of miRNAs targeting GIT1 indicated that there is good matching site for miR-125a-3p in the GIT1 3'UTR. A dual-luciferase reporter assay indicated that GIT1 is one of the target genes of miR-125a-3p. Therefore, in osteoblasts, miR-125a-3p may targetedly regulate GIT1 to play its role. Investigation of the BMP-2-induced differentiation process from HMSCs to osteoblasts indicates that miR-125a-3p expression increases gradually, but the level of increase is significantly suppressed compared with that of non-differentiating HMSCs, indicating that the inhibitory effect of miR-125a-3p expression is increasingly evident osteoblast differentiation proceeds. These results are similar to those of miR-125b reported by Mizuno et al (14), which indicates that miR-125a-3p may have a similar effect to miR-125b. miR-125b is the first reported miRNA to be associated with osteoblast differentiation (15). It was also found that 
the expression level of miR-125b decreased in the induction process of ST2 cells to osteoblasts, and after the introduction of miR-125b the expression level of ALP was lowered in the osteoblastic induction process of ST2 cells; by contrast, after adding miR-125b inhibitors the expression of ALP was enhanced (15). This indicates that miR-125b negatively regulates the osteoblastic differentiation process.

In the present study, as induced differentiation proceeded, the expression level of GIT1 increased significantly. Therefore, in the osteoblastic differentiation process, $\mathrm{miR}-125 \mathrm{a}-3 \mathrm{p}$ expression was negatively correlated with GIT1 expression; thus, an increase of GIT1 expression may be caused by suppressed miR-125a-3p expression. When miR-125a-3p mimics and inhibitors were each transfected into human osteoblasts, the findings were consistent with the speculation above. Specifically, when the miR-125a-3p expression level was changed, the expression level of GIT1 showed the opposite effect. This illustrates that miR-125a-3p targetedly regulates the expression of GIT1 in osteoblasts, and one of the pathways by which miR-125a-3p exerts its effects may involve the regulation of GIT1.

The impact of miR-125a-3p and GIT1 on osteoblastic proliferation and differentiation was determined in the present study, and the results indicated that miR-125a-3p targetedly regulates GIT1 expression to inhibit osteoblastic proliferation and differentiation. Feng et al (23) showed that miRNA-125a inhibited cell growth by targeting glypican-4 in the human embryonic kidney cell line 293T (HEK293T). Ninio-Many et al (24) found that miRNA-125a-3p reduced cell proliferation and migration by targeting Fyn in HEK293T cells. The study of Svensson et al (25) showed that inhibition of miRNA-125a promoted human endothelial cell proliferation and viability through an antiapoptotic mechanism. In addition, Guo et al (26) suggested that miRNA-125a repressed cell growth by targeting HuR in breast cancer. All the aforementioned findings are consistent with the conclusions of the present study, which both indicate a proliferation inhibiting effect of miR-125a-3p. In the study of Huang et al (27), the authors concluded that miR-125b was a key regulatory factor of osteoblastic differentiation that acted by directly targeting core binding factor $\beta$ and indirectly acting on Runx 2 at an early stage osteoblastic differentiation. Guo et al (28) reported that miR-125a plays a biological function in osteoclastogenesis through a novel TRAF6/NFATc1/miR-125a regulatory feedback loop, and that miR-125a was markedly downregulated during the macrophage colony stimulating factor- and receptor activator of nuclear factor- $\kappa \mathrm{B}$ ligand-induced osteoclastogenesis of circulating $\mathrm{CD}_{1} 4^{+}$peripheral blood mononuclear cells (PBMCs). Overexpression of miR-125a in CD14 ${ }^{+}$ PBMCs inhibited osteoclastogenesis, whereas the inhibition of miR-125a promoted osteoclastogenesis. Therefore, miR-125a-3p inhibits osteoblast proliferation and differentiation, and one of the pathways involves the regulation of GIT1.

In summary, miR-125a-3p affects osteoblast proliferation and differentiation through the regulation of GIT1. This finding increases our understanding of the physiological and pathological roles of miRNAs in osteoblastic differentiation and maturation processes, and should provide a theoretical basis for research into bone physiology and pathology. This may assist in the diagnosis and targeted treatment of metabolic bone disease and associated drug development.

\section{Acknowledgements}

This study was supported by Health Bureau of Wuxi City Foundation for Youths (grant no. Q201407).

\section{References}

1. Lemaire V, Tobin FL, Greller LD, Cho CR and Suva LJ: Modeling the interactions between osteoblast and osteoclast activities in bone remodeling. J Theor Biol 229: 293-309, 2004.

2. Alford AI, Kozloff KM and Hankenson KD: Extracellular matrix networks in bone remodeling. Int $\mathrm{J}$ Biochem Cell Biol 65: 20-31, 2015.

3. Arpornmaeklong P, Brown SE, Wang Z and Krebsbach PH: Phenotypic characterization, osteoblastic differentiation and bone regeneration capacity of human embryonic stem cell-derived mesenchymal stem cells. Stem Cells Dev 18: 955-968, 2009.

4. Schmalzigaug R, Phee H, Davidson CE, Weiss A and Premont RT: Differential expression of the ARF GAP genes GIT1 and GIT2 in mouse tissues. J Histochem Cytochem 55: 1039-1048, 2007.

5. Manabe R, Kovalenko M, Webb DJ and Horwitz AR: GIT1 functions in a motile, multi-molecular signaling complex that regulates protrusive activity and cell migration. J Cell Sci 115: 1497-1510, 2002.

6. Menon P, Yin G, Smolock EM, Zuscik MJ, Yan C and Berk BC: GPCR kinase 2 interacting protein 1 (GIT1) regulates osteoclast function and bone mass. J Cell Physiol 225: 777-785, 2010.

7. Ren Y, Yu L, Fan J, Rui Z, Hua Z, Zhang Z, Zhang N and Yin G: Phosphorylation of GIT1 tyrosine 321 is required for association with FAK at focal adhesions and for PDGF-activated migration of osteoblasts. Mol Cell Biochem 365: 109-118, 2012.

8. Bartel DP: MicroRNAs: Genomics, biogenesis, mechanism and function. Cell 116: 281-297, 2004.

9. Ambros V: The functions of animal microRNAs. Nature 431: 350-355, 2004.

10. Hassan MQ, Gordon JA, Beloti MM, Croce CM, van Wijnen AJ, Stein JL, Stein GS and Lian JB: A network connecting Runx2, SATB2 and the miR-23a 27a 24-2 cluster regulates the osteoblast differentiation program. Proc Natl Acad Sci USA 107: 19879-8435, 2010

11. Hu R, Li H, Liu W, Yang L, Tan YF and Luo XH: Targeting miRNAs in osteoblast differentiation and bone formation. Expert Opin Ther Targets 14: 1109-1120, 2010.

12. Kapinas K and Delany AM: MicroRNA biogenesis and regulation of bone remodeling. Arthritis Res Ther 13: 220, 2011.

13. Kapinas K, Kessler CB and Delany AM: miR-29 suppression of osteonectin in osteoblasts: Regulation during differentiation and by canonical Wnt signaling. J Cell Biochem 108: 216-224, 2009.

14. Mizuno Y, Yagi K, Tokuzawa Y, Kanesaki-Yatsuka Y, Suda T, Katagiri T, Fukuda T, Maruyama M, Okuda A, Amemiya T, et al: miR-125b inhibits osteoblastic differentiation by down-regulation of cell proliferation. Biochem Biophys Res Commun 368: 267-272, 2008.

15. Livak KJ and Schmittgen TD: Analysis of relative gene expression data using real-time quantitative PCR and the 2(-Delta Delta C(T)) Method. Methods 25: 402-408, 2001.

16. Scott GK, Goga A, Bhaumik D, Berger CE, Sullivan CS and Benz CC: Coordinate suppression of ERBB2 and ERBB3 by enforced expression of micro-RNA miR-125a or miR-125b. J Biol Chem 282: 1479-1486, 2007.

17. Meister G, Landthaler M, Patkaniowska A, Dorsett Y, Teng G and Tuschl T: Human Argonaute 2 mediates RNA cleavage targeted by miRNAs and siRNAs. Mol Cell 15: 185-197, 2004.

18. Chau JF, Leong WF and Li B: Signaling pathways governing osteoblast proliferation, differentiation and function. Histol Histopathol 24: 1593-1606, 2009.

19. Oskowitz AZ, Lu J, Penfornis P, Ylostalo J, McBride J, Flemington EK, Prockop DJ and Pochampally R: Human multipotent stromal cells from bone marrow and microRNA: Regulation of differentiation and leukemia inhibitory factor expression. Proc Natl Acad Sci USA 105: 18372-7, 2008. 
20. Wu Y, Zhang Y, Yin Q, Xia $\mathrm{H}$ and Wang J: Platelet-derived growth factor promotes osteoblast proliferation by activating G-protein-coupled receptor kinase interactor-1. Mol Med Rep 10: 1349-54, 2014.

21. Huang WC, Chan SH, Jang TH, Chang JW, Ko YC, Yen TC, Chiang SL, Chiang WF, Shieh TY, Liao CT, et al: miRNA-491-5p and GIT1 serve as modulators and biomarkers for oral squamous cell carcinoma invasion and metastasis. Cancer Res 74: 751-64, 2014

22. Chan SH, Huang WC, Chang JW, Chang KJ, Kuo WH, Wang MY, Lin KY, Uen YH, Hou MF, Lin CM, et al: MicroRNA-149 targets GIT1 to suppress integrin signaling and breast cancer metastasis. Oncogene 33: 4496-507, 2014.

23. Feng C, Li J, Ruan J and Ding K: MicroRNA-125a inhibits cell growth by targeting glypican-4. Glycoconj J 29: 503-11, 2012.

24. Ninio-Many L, Grossman H, Shomron N, Chuderland D and Shalgi R: microRNA-125a-3p reduces cell proliferation and migration by targeting Fyn. J Cell Sci 126: 2867-176, 2013.
25. Svensson D, Gidlöf O, Turczyńska KM, Erlinge D, Albinsson S and Nilsson BO: Inhibition of microRNA-125a promotes human endothelial cell proliferation and viability through an antiapoptotic mechanism. J Vasc Res 51: 239-245, 2014.

26. Guo X, Wu Y and Hartley RS: MicroRNA-125a represses cell growth by targeting HuR in breast cancer. RNA Biol 6: 575-583, 2009.

27. Huang K, Fu J, Zhou W, Li W, Dong S, Yu S, Hu Z, Wang H and Xie Z: MicroRNA-125b regulates osteogenic differentiation of mesenchymal stem cells by targeting $\operatorname{Cbf} \beta$ in vitro. Biochimie 102: 47-55, 2014.

28. Guo LJ, Liao L, Yang L, Li Y and Jiang TJ: MiR-125a TNF receptor-associated factor 6 to inhibit osteoclastogenesis. Exp Cell Res 321: 142-152, 2014. 